\title{
Exploring galactic wind superbubbles by multimessenger observations
}

\section{Enrico Peretti, ${ }^{a, *}$ Giovanni Morlino, ${ }^{b}$ Pasquale Blasi, ${ }^{c, d}$ Pierre Cristofari $^{e}$ and Markus Ahlers $^{a}$}

${ }^{a}$ Niels Bohr International Academy, Niels Bohr Institute,University of Copenhagen, Blegdamsvej 17, DK-2100 Copenhagen, Denmark

${ }^{b}$ INAF, Osservatorio Astrofisico di Arcetri, L.go E. Fermi 5, I-50125 Firenze, Italy

${ }^{c}$ Gran Sasso Science Institute, Viale F. Crispi 7, 67100 L’Aquila, Italy

${ }^{d}$ INFN/Laboratori Nazionali del Gran Sasso, Via G. Acitelli 22, 67100 Assergi (AQ), Italy

e Observatoire de Paris, PSL Research University, LUTH, 5 Place J. Janssen, 92195 Meudon, France

E-mail: peretti@nbi.ku.dk

Galactic winds are one of the most spectacular phenomena we observe in the Universe. They are common in active galaxies, and can be powered either by stellar feedback typical of star forming galaxies or by active galactic nuclei (AGN). These winds have a bubble structure characterized by an external forward shock expanding in the circumgalactic medium and an internal reverse shock separating the cool and fast wind from the hot shocked wind. While the forward shock is unlikely to be able to accelerate particles efficiently for a long time, at the reverse shock the necessary conditions for efficient acceleration may be present. The power of these outflows ranges from $10^{39} \mathrm{erg} \mathrm{s}^{-1}$ up to $10^{45} \mathrm{erg} \mathrm{s}^{-1}$ making galactic wind bubbles powerful particle accelerators. We develop a model for particle acceleration at the termination shock of such superbubbles analysing the differences between AGN-driven and starburst-driven scenarios. This is done solving the transport equation in the entire wind bubble structure accounting for diffusion, advection and energy losses. We show that the maximum energy in these systems can be of order $10^{2} \mathrm{PeV}$. We finally explore the associated multimessenger observables both in terms of escaping particles and hadronic byproducts such as gamma-rays and neutrinos produced via $\mathrm{pp}$ and $\mathrm{p} \gamma$ interactions.

$3^{\text {th }}$ International Cosmic Ray Conference (ICRC 2021)

July 12 th - 23rd, 2021

Online - Berlin, Germany

\footnotetext{
${ }^{*}$ Presenter
} 


\section{Introduction}

Outflows are ubiquitous in galaxies as they can be powered by several different processes such as stellar winds, supernovae or active galactic nuclei (AGN) [1]. In particular, starburst galaxies (SBGs) and AGN are characterized by an extreme release of energy in their central kiloparsec-scale region powered by an enhanced rate of supernovae (see [3]) and the accretion onto a super-massive black hole (SMBH, see [2]) respectively. The net result of such a strong energy release in a compact region is the growth of local temperature and pressure resulting eventually in a large-scale outflow expanding through the galactic halo. The power of the wind can range over several orders of magnitude, from $10^{39} \mathrm{erg} \mathrm{s}^{-1}$ up to $10^{45} \mathrm{erg} \mathrm{s}^{-1}$, making this system unique for particle acceleration and multimessenger emission [4-10].

The outflow is characterized by an onion-like structure similar to a bubble as shown in Fig. 1 and it is powered by a central engine, which is either an AGN or starburst nucleus (SBN). The wind breaking out the galactic disk is supersonic and expands adiabatically with approximately constant terminal velocity $\left(u_{1} \sim 10^{3} \mathrm{~km} \mathrm{~s}^{-1}\right.$, see also [11, 12]) in the galactic halo. The fast and cool wind is compressed and heated at the wind shock, which is indicated in the figure by $R_{\mathrm{sh}}$. The wind shock is a strong shock, where the Mach number can be expected to be as large as $\mathcal{M}_{\mathrm{sh}} \approx 10$, providing the conditions for efficient particle acceleration (see [10]). The shocked wind decelerates adiabatically $\left(u_{2} \propto r^{-2}\right)$ up to the contact discontinuity separating the wind fluid from the shocked halo medium. Ahead of the contact discontinuity one can find the forward shock indicated in the figure by $R_{\mathrm{FS}}$. The forward shock is expected to be mildly transonic, $\mathcal{M}_{\mathrm{FS}} \approx 1$, limiting the efficiency of particle acceleration at such discontinuity.

The dynamics of the wind bubble is characterized by an initial free expansion during which the two shocks and the contact discontinuity expand very close to each other. As soon as the swept up mass becomes comparable with the mass of the wind itself the system decelerates. At this point the evolution of the system becomes self-similar and the two shocks decouple. In particular, in a constant density ambient medium, the wind shock slows down its expansion as $R_{\mathrm{sh}} \sim t^{2 / 5}$ while the forward shock proceeds as $R_{\mathrm{FS}} \sim t^{3 / 5}[12,13]$. The decoupling of the shocks results in an expansion of the shocked wind region which starts inflating. At late times, when the pressure in the shocked bubble has decreased to a level comparable with the halo pressure $\left(P_{h}\right)$, the wind shock can stall while being sustained by the cool wind ram pressure, So that $R_{\mathrm{sh}} \approx \sqrt{\dot{M} u_{1} /\left(4 \pi P_{h}\right)}$.

\section{Particles and radiation in the wind bubble}

The evolution of the system is slow compared to the typical timescales of particle acceleration, so that stationary conditions allow to characterize the population of high-energy particles in the system. The innermost part of the system is described by a wind radial profile $u_{1}(r)$ and particle density $n_{1}(r)$ that can be derived following the prescriptions of [11] once the main parameters of the wind, such as the mass-loss-rate $\dot{M}$ and the terminal wind speed $V_{\infty} \approx u_{1}$, are set. The shocked wind is characterized by the radial velocity profile $u_{2}(r) \propto r^{-2}$ and a constant density. The wind superbubble is approximately spherical, so that the transport of particles is treated assuming spherical symmetry. We estimate the magnetic field strength in the system assuming that a fraction $\varepsilon_{\mathrm{B}} \sim 10 \%$ of the kinetic energy density of the upstream fluid, $m_{p} n u_{1}^{2} / 2$, is converted into magnetic 


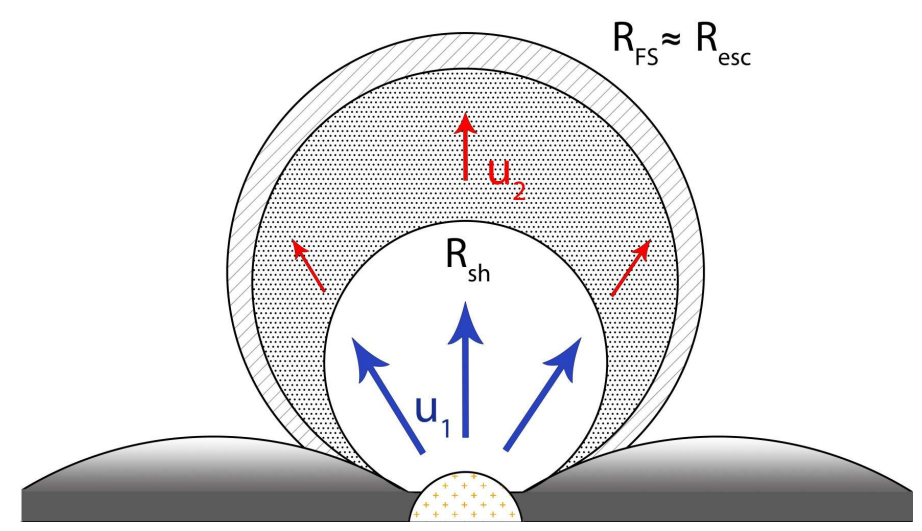

Figure 1: Wind bubble structure. The central source (starburst nucleus or AGN), from which the wind is launched, is located in the center of the galactic disk. The blue (red) arrow corresponds to the cool (shocked) wind region. The wind shock $\left(R_{\mathrm{sh}}\right)$ separates the two regions. The forward shock (at $\left.R_{\mathrm{FS}}\right)$ corresponds to the boundary of the system from the undisturbed halo region (credit: I. Peretti).

field energy density $U_{B}=B^{2} / 8 \pi$. We also assume that at the wind shock the perpendicular components of the magnetic field are compressed by a factor 4 , which implies that the strength of the magnetic field is enhanced by a factor $\sqrt{11}$ and remains spatially constant in the whole downstream region.

In this context, we model particle acceleration and transport of energetic protons by solving the CR transport equation in the entire wind superbubble. Particles are injected at the wind shock position $R_{\mathrm{sh}}$ and freely escape once they reach the forward shock at $R_{\mathrm{FS}}$. Particles in the system are described by

$$
u(r) \nabla f(r, p)=\nabla[D(r, p) \nabla f(r, p)]+\frac{p}{3} \partial_{p} f(r, p) \nabla u(r)+Q(r, p)-L(r, p) .
$$

Here $f$ is the CR phase space density depending on the radial coordinate $r$ and momentum $p$ of particles. We assume particle injection at the wind shock, $Q(r, p)=\eta u_{1} n_{1} /\left[4 \pi p^{2}\right] \delta\left[p-p_{\text {inj }}\right] \delta[R-$ $R_{\text {sh }}$ ], where $\eta<1$ is an efficiency factor, and $L(r, p)=n(r) \sigma_{p p}(p) c f(r, p)$ is the energy loss term accounting for $p p$ collisions. The space-dependent diffusion coefficient is assumed to follow, $D(r, p)=r_{\mathrm{L}}(r, p)^{2-\delta} l_{c}^{\delta-1} / 3$, motivated by quasi-linear theory where $r_{\mathrm{L}}$ is the Larmor radius and $l_{c}$ is the coherence length of the turbulent magnetic field. We adopt $\delta=1.5$ as prescribed by MHD (Kraichnan) turbulence and we assume $l_{c}=10^{2} \mathrm{pc}$. We also account for a transition of the diffusion regime for momenta larger than a critical momentum scale $p_{*}$ determined by $r_{\mathrm{L}}\left(p_{*}\right)=l_{c}$. Following Ref. [15], we assume $D(p)=D\left(r, p_{*}\right)\left(p / p_{*}\right)^{2}$ for $p>p_{*}$.

We solve the transport equation following the procedure developed in [14] and adopted by [10] in the context of starbursts. We refer the interested reader to these works for additional details on the numerical solutions and analytic expressions of the functions introduced below. In the following we limit ourselves to reporting and discussing the main physical insight of the solution at the shock, in the internal cool wind (upstream) and in the outer warm shocked wind (downstream). The solution at the shock has the form

$$
f_{\mathrm{sh}}(p)=\frac{s n_{1} \eta_{\mathrm{CR}}}{4 \pi p_{\mathrm{inj}}^{3}}\left(\frac{p_{\mathrm{inj}}}{p}\right)^{s} e^{-\left[\Gamma_{1}(p)+\Gamma_{2}(p)\right]},
$$



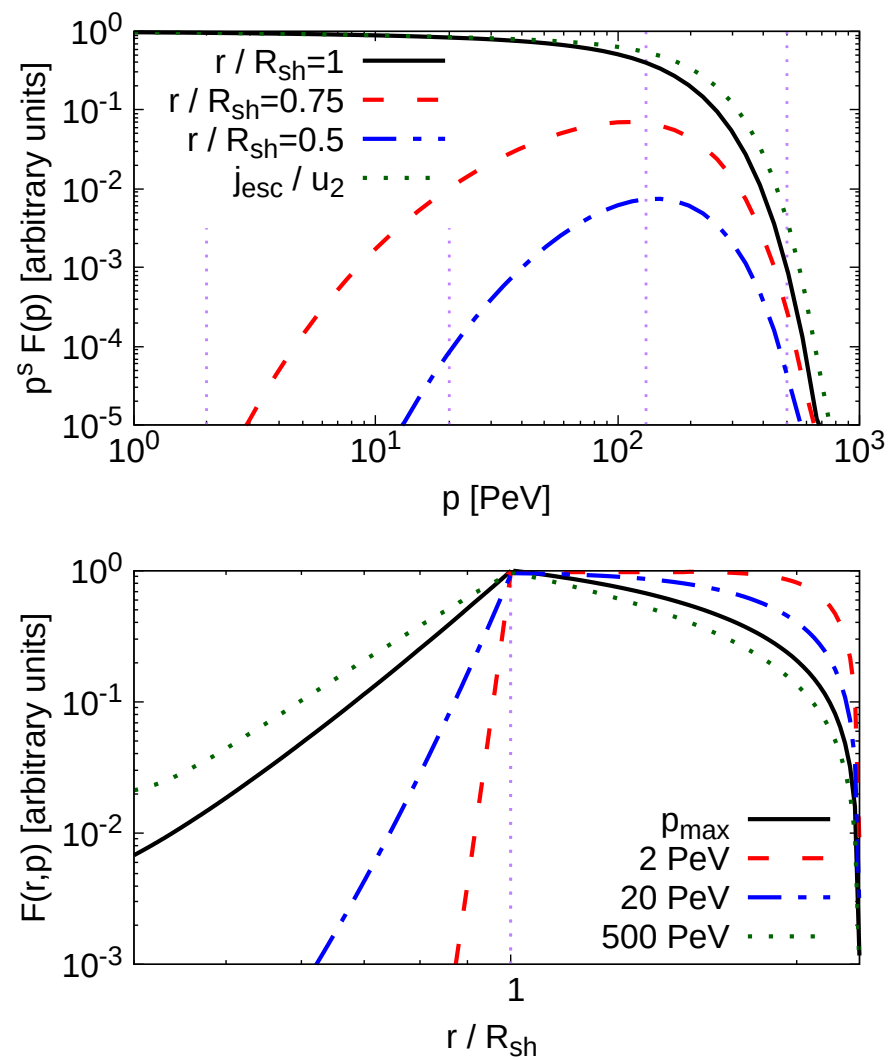

Figure 2: Particle spectrum in momentum space and in physical space. Top panel: Particle spectrum as a function of momentum. We show the solution at the shock (thick black line) compared to the solution at $0.75 R_{\mathrm{sh}}$ (red dashed line) and $0.5 R_{\mathrm{sh}}$ (blue dot-dashed line). The escaping flux is indicated by the green dotted line. The vertical violet dotted lines highlight the energies at which the radial behavior is shown in the bottom panel. Bottom panel: Radial scaling of the solution at different energies highlighted in the top panel. We show the solution at $p_{\max }(131 \mathrm{PeV}$, thick black line) compared with the solution at $2 \mathrm{PeV}$ (dred dashed line), $20 \mathrm{PeV}$ (dot-dashed line) and $500 \mathrm{PeV}$ (green dotted). The vertical dotted line indicates the shock position.

where one can recognize a power-law behavior with index $s$ in agreement with the standard prediction of diffusive shock acceleration (DSA). The effective high-energy cut off and its shape is determined by the functions $\Gamma_{1}$ and $\Gamma_{2}$ which account for the geometry and energy losses in the upstream and downstream regions respectively. The acceleration efficiency $\eta_{\mathrm{CR}}$ is taken such that the total CR pressure at the shock is $10 \%$ of the fluid ram pressure $\left(P_{\text {ram }}=n m_{p} u_{1}^{2}\right)$. The solution $f_{1}$ in the upstream region is regulated by the competition between diffusion and advection as

$$
f_{1}(r, p)=f_{\mathrm{sh}}(p) e^{-\int_{r}^{R_{\mathrm{sh}}} d r^{\prime} V_{1}\left(r^{\prime}, p\right) / D_{1}\left(r^{\prime}, p\right)}
$$

where $V_{1}$ is the effective advection speed experienced by particles at different momenta. Particularly interesting is the spatial behavior of the solution, which is exponentially suppressed according to the effective diffusion length $\lambda_{D}=D_{1} / V_{1}$. The solution $f_{2}$ in the downstream region is set by the dominant role of the advection over diffusion and the escape taking place at $R_{\mathrm{FS}}=R_{\mathrm{esc}}$, whereas 
energy losses are not dynamically relevant. Its analytic expression follows

$$
f_{2}(r, p)=f_{\mathrm{sh}}(p) \frac{1-e^{\alpha(r, p)-\alpha\left(R_{\mathrm{esc}}, p\right)}}{1-e^{-\alpha\left(R_{\mathrm{esc}}, p\right)}},
$$

where the parameter $\alpha$ contains the physical information on CR transport and escape, $\alpha(r, p)=$ $\left[R_{\mathrm{sh}} u_{2} / D_{2}(p)\right]\left(1-R_{\mathrm{sh}} / r\right)$. The escaping flux is defined as $j_{\mathrm{esc}}=-D \partial_{r} f$ evaluated at the free escape boundary $R_{\text {esc }}$. Its expression is analytic and reads

$$
j_{\mathrm{esc}}(p)=u_{2} f_{\mathrm{sh}}(p)\left[R_{\mathrm{sh}} / R_{\mathrm{esc}}\right]^{2} /\left[1-e^{-\alpha\left(R_{\mathrm{esc}}, p\right)}\right] .
$$

Figure 2 shows the spectrum of accelerated particles in momentum space (top panel) and physical space (bottom panel). These results are obtained in a prototype scenario (hereafter PS1) characterized by the following set of parameters: $\dot{M}=10 \mathrm{M}_{\odot} \mathrm{yr}^{-1}, u_{1}=3000 \mathrm{~km} \mathrm{~s}^{-1}$ and $P_{h} / k_{\mathrm{B}} \approx 5 \cdot 10^{4} \mathrm{~K} \mathrm{~cm}^{-3}$. The age of the system is assumed to be $t_{\text {age }}=10^{2} \mathrm{Myr}$, so that $R_{\mathrm{sh}} \approx 15 \mathrm{kpc}$ while $R_{\text {esc }} \approx 35 \mathrm{kpc}$. By defining $p_{\max }$ as the momentum at which $p^{s} f(p)$ is reduced by one efolding with respect to the extrapolation at high momenta of $p^{s} f\left(p_{\text {inj }}\right)$, we find that particles can be accelerated to energies as large as $10^{2} \mathrm{PeV}$ in this system. The shape of the spectrum at the shock (top panel, thick black curve) agrees with the standard DSA prediction, $p^{-s}$, for $p \ll p_{\max }$, while the transport solution at smaller radii presents a strong low-energy cut off (top panel, red dashed and blue dot-dashed curves). Complementary information is provided by the shape of the distribution function in physical space (bottom panel), where one can appreciate the competition between advection, which pushes particles towards the shock, and diffusion, which tries to isotropize the spatial distribution. This competition is encapsulated in the momentum-dependent diffusion length $\lambda_{D}$, which allows only particles with $p \approx p_{\max }$ to approach the center of the system while suppressing the back-streaming of particles with low momenta. The solution in the shocked wind region (downstream) is dominated by advection. Therefore, the escaping flux (top panel, green dotted curve) is almost identical to the particle spectrum at the shock. The dominant advection can also be observed in the spatial solution (bottom panel), where the particle density is practically flat.

Gamma rays $(\gamma)$ and neutrinos $(v)$ are produced in both the upstream and downstream regions as the result of $p p$ and $p \gamma$ interactions, where we assume that the target photon field is the lowenergy spectral energy distribution (SED) of NGC253 described in [16]. We compute the emissivity in gamma-rays as the result of $p p$ collisions following the prescription of [17] and adopting the NAIMA package [18]. The flavor-averaged neutrino luminosity is then obtained adopting the scaling $L_{v}\left(E_{v}\right) \approx L_{\gamma}\left(E_{\gamma}\right) / 2$, where $E_{\gamma} \approx 2 E_{\nu}$. The $\gamma$ and $v$ emissivity resulting from $p \gamma$ interactions is obtained adopting the formalism presented in [19]. Catastrophic energy losses such as $p p$ and $p \gamma$ do not have a relevant dynamical impact on the accelerated particles because the plasma is highly diluted in the wind bubble and the radiation field decays as $r^{-2}$ from the central source. Despite of the low dynamical relevance, particles are confined in the wind bubble system for a sufficiently long time to sustain high luminosity, especially coming from the downstream region where the solution is spatially flat.

Figure 3 shows the gamma-ray (thick red line) and neutrino (blue dot-dashed and orange double-dot-dashed for $p p$ and $p \gamma$, respectively) SED obtained for the parameter set PS1 assuming a source distance of $3.9 \mathrm{Mpc}$. The results are compared with the gamma-ray (green dashed) and neutrino (pink dotted) emission of a starburst nucleus (SBN) similar to NGC253 (see [20]), 


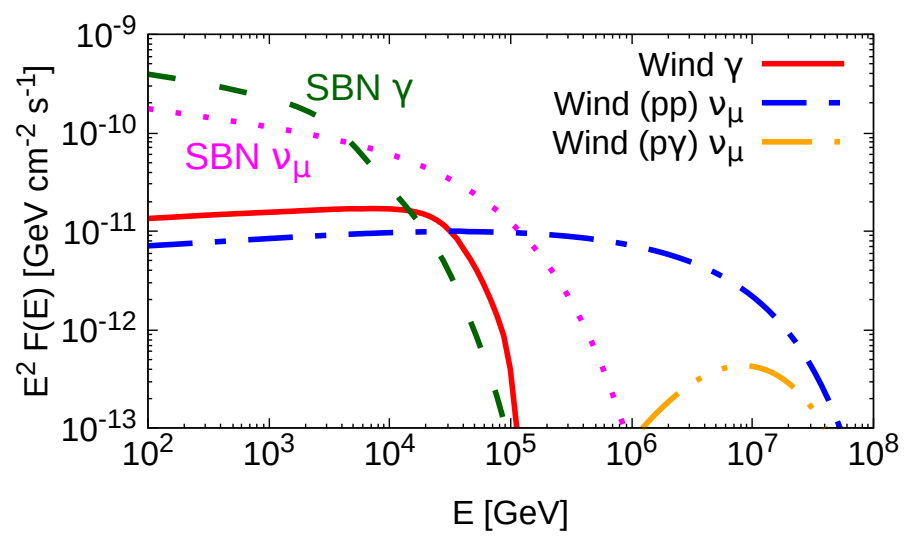

Figure 3: High-energy gamma-ray and neutrino spectra at Earth assuming a luminosity distance $d_{L}=$ 3.9 Mpc for the same accelerated particle population shown in Fig. 2. We show the gamma-ray and neutrino flux from the wind (thick red and dot-dashed blue lines) compared to the emission from an hypothetical coexisting SBN (green dashed and pink dotted lines) tuned to the properties of the starburst nucleus of NGC253 as described in [20] and assuming $p_{\max , \mathrm{SBN}}=1 \mathrm{PeV}$. The effect of EBL absorption on the gamma-ray spectrum is also taken into account.

where the maximum energy is assumed to be $1 \mathrm{PeV}$ as inferred for accelerators in the Milky Way. In contrast to the emission of the host galaxy, gamma rays produced in the wind bubble suffer only absorption in the cosmic microwave background (CMB) and in the extragalactic background light (EBL, see [21]), possibly extending the unabsorbed spectrum up to $\gtrsim 30 \mathrm{TeV}$. The neutrino emission is dominated by the wind bubble emission at $\sim 10^{2} \mathrm{TeV}$ where the high-energy cut off of protons accelerated by galactic sources is reflected in the secondary byproducts.

\section{Deciphering maximum energy and luminosity}

The power of galactic winds can range over several orders of magnitude $\left(10^{39}-10^{45} \mathrm{erg} \mathrm{s}^{-1}\right)$ leading to a large variety of objects in terms of maximum energy of accelerated particles and luminosity. We explore different realizations of these systems by performing a scan of the parameter space regulating the total power under the assumption of constant halo pressure $P_{h} / k_{\mathrm{B}}=5$. $10^{4} \mathrm{~K} \mathrm{~cm}^{-3}$ and $10^{2} \mathrm{Myr}$ as typical age of the system. In particular, we allow $\dot{M}$ to range from $0.1 \mathrm{M}_{\odot} \mathrm{yr}^{-1}$ up to $50 \mathrm{M}_{\odot} \mathrm{yr}^{-1}$, and $u_{1}$ from $500 \mathrm{~km} \mathrm{~s}^{-1}$ up to $3000 \mathrm{~km} \mathrm{~s}^{-1}$. We focus on the maximum energy of accelerated particles and the bubble luminosity in terms of flavor-averaged neutrino luminosity at $25 \mathrm{TeV}\left(L_{v_{\mu}}(25 \mathrm{TeV})\right)$. In the left panel of Fig. 4 we report the color-coded scatter-plot associated to $p_{\max }$, while in the right panel we show the corresponding values for $L_{v_{\mu}}(25 \mathrm{TeV})$. The maximum energy of accelerated particles extends over more than three orders of magnitude, from $\lesssim 0.1 \mathrm{PeV}$ up to $\gtrsim 10^{2} \mathrm{PeV}$, and increases with increasing power of the system. In general, it is possible to observe that mild wind powers $\left(\sim 10^{41} \mathrm{erg} \mathrm{s}^{-1}\right)$ are sufficient to accelerate particles at energies at least $\gtrsim \mathrm{PeV}$. The bubble luminosity, parametrized by $L_{\nu_{\mu}}(25 \mathrm{TeV})$, has a less trivial behavior because it depends on the convolution of the target density with the size of the downstream region where most of the emission takes place. The latter is determined by the dynamics of the system and its age. In our scenario we are keeping the age and the external pressure 

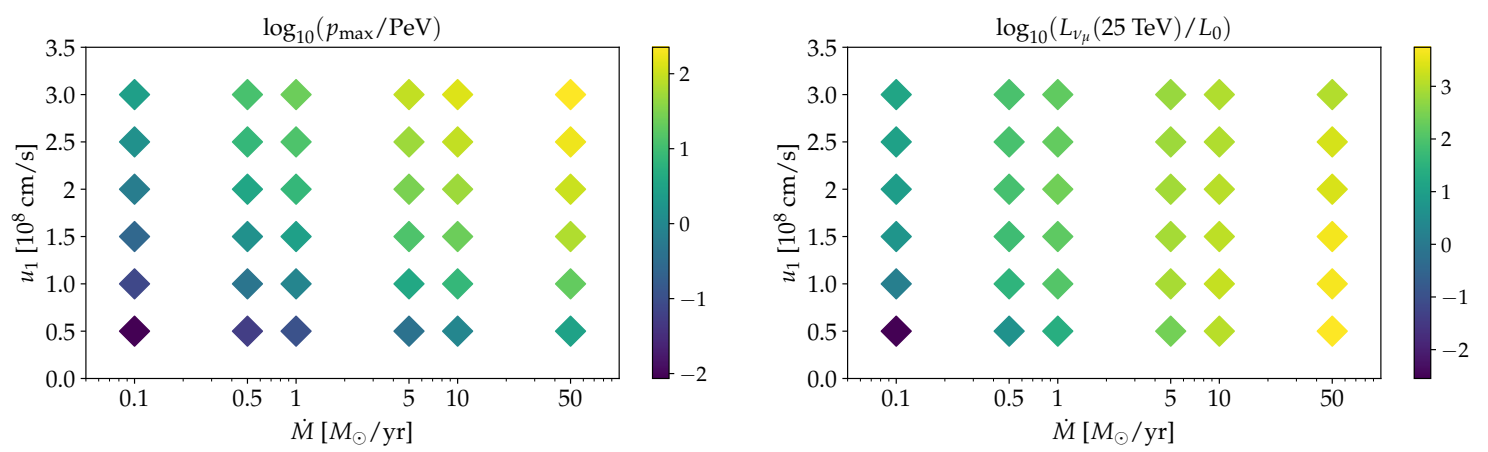

Figure 4: Scatter plots of $p_{\max }$ (left panel) and $L_{\nu_{\mu}}(25 \mathrm{TeV})$ (right panel) for different configurations of $\dot{M}$ and $u_{1}$. The luminostiy $L_{\nu_{\mu}}(25 \mathrm{TeV})$ is normalized to the nominal luminosity $L_{0} \approx 4.7 \cdot 10^{3} \mathrm{~L}_{\odot}$.

constant. Therefore the luminosity grows with increasing wind power provided that the shocked wind region has enough time to inflate. For this reason $L_{v_{\mu}}(25 \mathrm{TeV})$ mildly increases for higher wind speed up to $\dot{M} \sim 10 \mathrm{M}_{\odot} \mathrm{yr}^{-1}$. For higher $\dot{M}$ we obtain the opposite behavior since the shocks characterizing the system decouple at a time comparable with the age of the system.

We finally explored more powerful systems with different conditions of external halo pressure. While starburst winds are unlikely to reach velocities higher than $\sim 3000 \mathrm{~km} \mathrm{~s}^{-1}$, winds with extreme mass loss rates, such as luminous infrared galaxies (LIRG and ULIRG), or outflows driven by AGN can be responsible for higher powers. [22]. In particular, we found that galactic wind superbubbles characterized by velocities $\gtrsim 5000 \mathrm{~km} \mathrm{~s}^{-1}$ and $P_{h} / k_{\mathrm{B}} \approx 5 \cdot 10^{5} \mathrm{~K} \mathrm{~cm}^{-3}$ can accelerate particles at energies as high as $\sim \mathrm{EeV}$ at their wind termination shocks.

\section{Summary and conclusions}

We explored particle acceleration and multimessenger emission in galactic wind superbubbles. We found that mild winds $\left(\gtrsim 10^{41} \mathrm{erg} \mathrm{s}^{-1}\right.$ ) are likely sufficient to accelerate particles to PeV energies while stronger winds $\left(\gtrsim 10^{43} \mathrm{erg} \mathrm{s}^{-1}\right.$ ) are necessary to reach $10^{2} \mathrm{PeV}$. Cosmic ray reaching EeV energies require systems powered by extreme injection power $10^{44}-10^{45} \mathrm{erg} \mathrm{s}^{-1}$. These activity levels are unlikely to be found in SBGs but can be found in LIRG, ULIRG and AGN. Wind superbubbles have a great potential as multimessenger sources in terms of escaping cosmic rays, and hadronic byproducts such as gamma rays and neutrinos. In particular, systems with longer lifetime are expected to be more luminous in gamma rays and neutrinos due to the expansion of the system. Wind superbubbles powered by SBGs and AGN are promising candidates for future investigations of both point-like and diffuse radiation of gamma rays, neutrinos and CRs at the highest energies (see also [6, 10, 23, 24]).

\section{Acknowledgments}

EP is grateful to Alessandra Lamastra for several insightful discussions. The research activity of EP and MA was partially supported by Villum Fonden (project n. 18994). EP was also partially supported by the European Union's Horizon 2020 research and innovation program under the Marie Sklodowska-Curie grant agreement No. 847523 'INTERACTIONS'. 


\section{References}

[1] Veilleux S., et al., 2005, ARA\&A, 43, 769. doi:10.1146/annurev.astro.43.072103.150610

[2] King A., Pounds K., 2015, ARA\&A, 53, 115. doi:10.1146/annurev-astro-082214-122316

[3] Zhang D., 2018, Galax, 6, 114. doi:10.3390/galaxies6040114

[4] Lamastra A., et al., 2016, A\&A, 596, A68. doi:10.1051/0004-6361/201628667

[5] Lamastra A., et al., 2019, APh, 112, 16. doi:10.1016/j.astropartphys.2019.04.003

[6] Liu R.-Y., et al., 2018, ApJ, 858, 9. doi:10.3847/1538-4357/aaba74

[7] Romero G. E., et al., 2018, A\&A, 616, A57. doi:10.1051/0004-6361/201832666

[8] Müller A. L., Romero G. E., Roth M., 2020, MNRAS, 496, 2474. doi:10.1093/mnras/staa1720

[9] Bustard C., Zweibel E. G., Cotter C., 2017, ApJ, 835, 72. doi:10.3847/1538-4357/835/1/72

[10] Peretti E., Morlino G., Blasi P., Cristofari P., 2021, arXiv, arXiv:2104.10978

[11] Chevalier R. A., Clegg A. W., 1985, Natur, 317, 44. doi:10.1038/317044a0

[12] Koo B.-C., McKee C. F., 1992, ApJ, 388, 93. doi:10.1086/171132

[13] Weaver R.,et al., 1977, ApJ, 218, 377. doi:10.1086/155692

[14] Morlino G., Blasi P., Peretti E., Cristofari P., 2021, MNRAS.tmp. doi:10.1093/mnras/stab690

[15] Subedi P., et al., 2017, ApJ, 837, 140. doi:10.3847/1538-4357/aa603a

[16] Galliano F., Dwek E., Chanial P., 2008, ApJ, 672, 214. doi:https://doi.org/10.1086/523621

[17] Kelner S. R., et al., 2006, PhRvD, 74, 034018. doi:10.1103/PhysRevD.74.034018

[18] Kafexhiu E., et al., 2014, PhRvD, 90, 123014. doi:10.1103/PhysRevD.90.123014

[19] Kelner S. R., Aharonian F. A., 2008, PhRvD, 78, 034013. doi:10.1103/PhysRevD.78.034013

[20] Peretti E., et al., 2019, MNRAS, 487, 168. doi:10.1093/mnras/stz1161

[21] Franceschini A., Rodighiero G., 2017, A\&A, 603, A34. doi:10.1051/0004-6361/201629684

[22] Fiore F., et al., 2017, A\&A, 601, A143. doi:10.1051/0004-6361/201629478

[23] Peretti E., et al., 2020, MNRAS, 493, 5880. doi:10.1093/mnras/staa698

[24] Lamastra A., et al., 2017, A\&A, 607, A18. doi:10.1051/0004-6361/201731452 